\title{
Eyes are special but not for everyone: The case of autism
}

\author{
Jelena Ristic $^{\mathrm{a}, *}$, Laurent Mottron ${ }^{\mathrm{b}}$, Chris Kelland Friesen ${ }^{\mathrm{c}}$, Grace Iarocci ${ }^{\mathrm{d}}$, \\ Jacob A. Burack ${ }^{\mathrm{e}}$, Alan Kingstone ${ }^{\mathrm{a}}$ \\ ${ }^{a}$ Department of Psychology, University of British Columbia, 2136 West Mall, Vancouver, BC, Canada V6T $1 Z 4$ \\ ${ }^{\mathrm{b}}$ University of Montreal, Hôpital Rivière-des-Prairies, Canada \\ ${ }^{\mathrm{c}}$ North Dakota State University, Fargo, ND 58105, USA \\ ${ }^{\mathrm{d}}$ Simon Fraser University, Canada \\ ${ }^{\mathrm{e}}$ McGill University, Hôpital Rivière-des-Prairies, Canada
}

Accepted 10 February 2005

Available online 17 March 2005

\begin{abstract}
Current research indicates that human gaze direction is a special cue for shifting attention for one of two reasons: (1) it reflects social desires and intentions and (2) its basic perceptual features usually correspond to important events in the environment. This study, conducted with individuals with autism and with age- and IQ-matched typically developing individuals, dissociates these two often-confounded explanations and demonstrates that eyes appear to be special for typically developing individuals because of their social power, whereas gaze effects are mediated by feature correspondence among persons with autism.
\end{abstract}

(C) 2005 Elsevier B.V. All rights reserved.

Theme: Neural basis of behavior

Topic: Cognition

Keywords: Eye direction; Social attenuation; Autism

Why do we have a tendency to shift our attention to where other people are looking? Investigations suggest that there are two possible explanations. One is that eye direction conveys key social information, such as status, personal interest, and attentional engagement [2]. We call this the social reading hypothesis. The other is that people are sensitive to changes in the basic stimulus features that are associated with shifts in gaze direction, in particular, the correspondence between the location of an interesting event in the environment and the position of the pupils/irises in the eyes that are directed towards that location $[6,13]$. We call this the feature correspondence hypothesis.

In the past, these two conceptualizations were tied so closely to one another that they were often discussed as

\footnotetext{
* Corresponding author. Fax: +1 6048220069.

E-mail address: jelena.ristic@telus.net (J. Ristic).
}

though they were synonymous, as it is difficult to imagine a natural situation in which the social meaning associated with gaze direction and the perceptual features associated with gaze direction could be disentangled [5]. In the present study, we show that the two indeed can be dissociated, a finding that carries substantial implications for the understanding of human social cognition.

We examined the performance of a total of 47 participants (see Table 1), who viewed static displays of left- and right-deviated gaze on a computer screen. The participants were asked to make a speeded keypress response when they detected a target occurring to the left or right of the face following one of four gaze cue-target delay intervals (see Fig. 1). Both high functioning individuals with autism (HFA) and typically developing peers (TD) were assigned randomly to either the nonpredictive gaze condition or the predictive gaze condition. In the nonpredictive condition, a target appeared at the gazed-at location $50 \%$ of the time and 


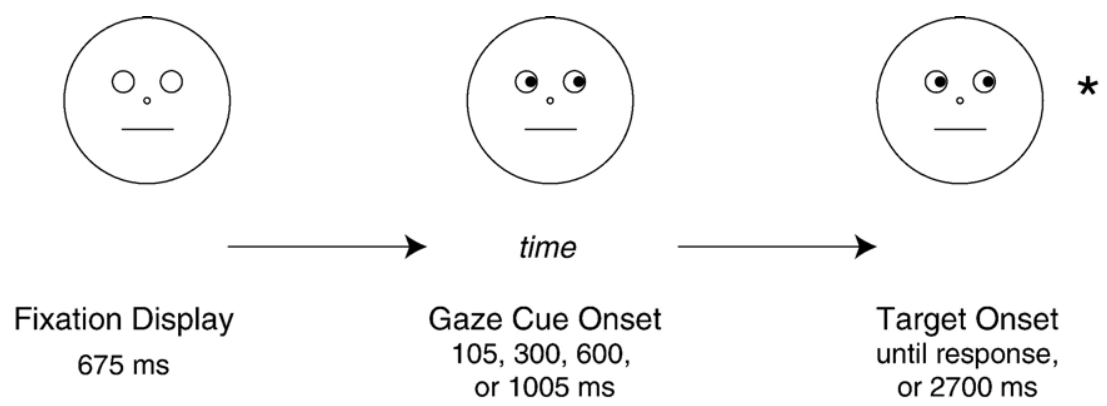

Fig. 1. Illustration (not to scale) of stimuli and sample sequence of events. The start of each trial was signaled by the presentation of a schematic face with blank eyes. The pupils, looking left or right, appeared $675 \mathrm{~ms}$ later. A target presented at an eccentricity of $3.55^{\circ}$ of visual angle was shown on the left or the right side of the fixation stimulus 105, 300, 600, or $1005 \mathrm{~ms}$ after appearance of the pupils (the attentional cue). Both the central face and the target remained on the screen until a response was made or 2, $700 \mathrm{~ms}$ had elapsed, whichever came first. Speeded response time was measured from the onset of the target. Participants were instructed to maintain central fixation and were informally monitored as it is well established that eye movements do not occur when suprathreshold targets such as those used here must be detected [7]. In the nonpredictive cue condition, each participant received a total of 336 experimental trials, divided equally over 8 testing blocks while in the predictive cue condition each participant received 672 trials, divided equally over 16 testing blocks.

High Functioning Autism

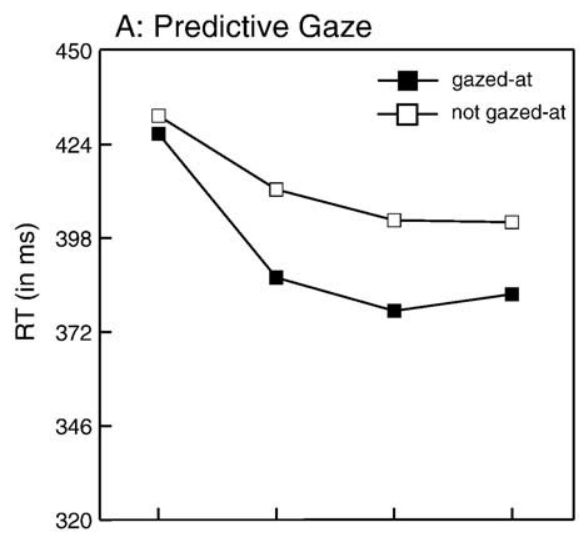

B: Nonpredictive Gaze

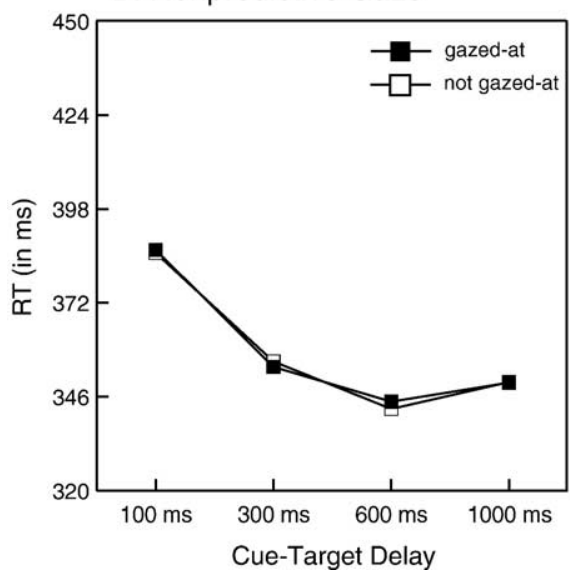

Typically Developing

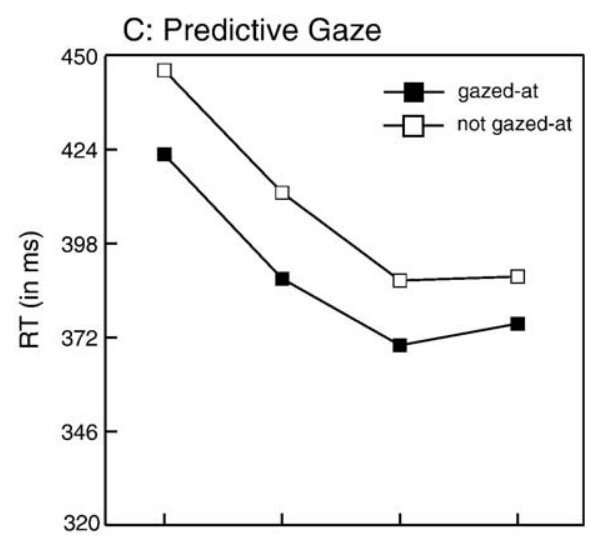

D: Nonpredictive Gaze

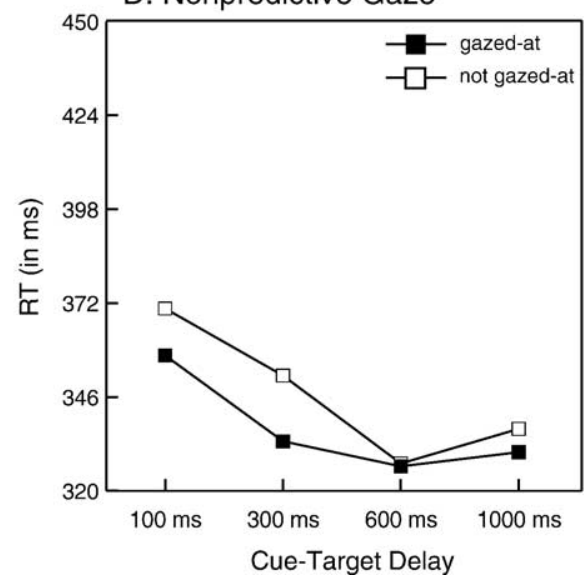

Fig. 2. Mean response time (RT) in milliseconds (ms) for high-functioning individuals with autism (HFA) and typically developing (TD) individuals as a function of gaze cue-target delay when the target appeared at a location that was or was not gazed-at. The column on the left shows HFA performance when gaze direction was predictive (top figure) and when it was nonpredictive (bottom figure) of target location. The column on the right shows TD performance when gaze was predictive (top figure) and when it was nonpredictive (bottom figure) of target location. Mean response error never exceeded 3.6\% and averaged $0.9 \%$. An analysis of variance (ANOVA) of HFA RT performance in the predictive condition, with gaze-target stimulus onset asynchrony (SOA) and gaze-target validity as factors, revealed significant main effects for both SOA and gaze validity [both $F$ 's $>8.5, P$ 's $<0.01$ ] with the validity effect growing as a function of SOA $[F(3,33)=4.52, P<0.01]$. In the nonpredictive condition, there was no effect for gaze direction and no interaction between gaze and SOA [both $F$ 's $<1]$ although there was a main effect of SOA $[F>35, P<0.05]$. A between-subjects ANOVA comparing the two conditions confirmed that there was a significant gaze $\times$ predictiveness interaction $[F(1,22)=11.23, P<0.01]$. The same set of analyses conducted on the TD data revealed that for the predictive condition, and for the nonpredictive condition, all main effects were significant [all $F$ 's $>10.4, P<0.01$ ] as were the gaze $\times$ SOA interactions [both $F$ 's $>2.93, P<0.05]$. A between-subjects ANOVA comparing the two conditions revealed that the gaze $\times$ predictiveness interaction was not significant $[F(1,21)=1.0, P>0.3]$. 
Table 1

Detailed participant information

\begin{tabular}{lllll}
\hline Condition & Group & $N$ & Mean IQ & Mean CA \\
\hline Predictive gaze & HFA & 12 (11 males) & 106.1 & 17.1 \\
& TD & 11 (7 males) & 100.1 & 15.0 \\
Nonpredictive gaze & HFA & 12 (12 males) & 110.8 & 20.5 \\
& TD & 12 (12 males) & 114.8 & 21.8 \\
\hline
\end{tabular}

All individuals included in High Functioning Autism (HFA) group met the diagnostic criteria for HFA or Asperger Syndrome according to the Autism Diagnostic and Observational Schedule-Generic [15] and the Autism Diagnostic Interview-Revised [14]. Participants included in the typically developing (TD) group were screened for history of psychiatric disorders. Groups were matched for mean IQ and chronological years of age (CA). Four participants (not shown) were excluded due to failures to perform the speeded aspect of the task.

at the not-gazed-at location $50 \%$ of the time. In the predictive condition, a target appeared at the gazed-at location $80 \%$ of the time and at the not-gazed-at location $20 \%$ of the time.

When eye direction was spatially predictive, as illustrated in Figs. 2A and C, both HFAs and TDs were faster to detect targets occurring at the gazed-at location. This indicates that both groups could perceive and use gaze direction as an attentional cue when the correspondence information was known to be a reliable aid to the task at hand. The key question, however, is whether attending to a gazed-at location is driven by the stimulus features (high correspondence between the cue and the target) or by social relevance of perceived gaze direction.

Figs. 2B and D show that, consistent with previous findings $[6,16]$, TDs shifted their attention in response to perceived eye direction when it was spatially nonpredictive (that is, when the correspondence between eye direction and target location was at chance). In contrast, HFAs did not shift their attention in response to nonpredictive eye direction. This difference in performance supports the social reading hypothesis as TDs, who can respond to the social power of eyes, orient automatically in response to gaze direction even when it conveys no predictive information about environmental events. In contrast, HFAs do not attend to eye direction when it is spatially nonpredictive. This is consistent with the notion that HFAs are not sensitive to eyes as displaying socially relevant information $[8,10,18]$, but are exquisitely sensitive to changes in event probability in their environment [11]. Thus, our experiment supports the feature correspondence hypothesis with regard to HFAs. It also highlights the outcome that HFAs essentially outperform the TDs in the nonpredictive condition insofar as HFAs were not "fooled" by a nonpredictive gaze cue.

The results of the present study suggest that efforts to train individuals with autism to use eye direction as a probabilistic feature correspondence cue fail to capture the key and fundamental component that gaze direction is normally used as a cue that is prioritized by the human attention system because of its social relevance. At best, individuals with autism appear to learn to orient attention to features that are usually confounded with eye direction, such as abrupt transients and stimulus motion [3,12,17,19]. This failure to appreciate the social power of human eyes appears to be grounded in fundamental differences in brain function between individuals with autism and the general population. For example, functional neuroimaging evidence indicates that the superior temporal sulcus' typical specialization for processing faces and eyes $[1,4,9]$ is not evident consistently in individuals with autism $[10,18]$.

Our study provides evidence that perceived social relevance, and not feature correspondence, drives automatic attentional orienting in response to gaze direction for typically developing individuals, but that feature correspondence, and not social relevance, mediates attention to gaze effects in individuals with autism. As such, the present study provides the first dissociation of these two oftenconfounded explanations, carrying with it important implications for understanding the development of social attention in both healthy and atypical populations.

\section{Acknowledgments}

Supported by graduate student awards to JR from the Natural Sciences and Engineering Research Council (NSERC) and the Michael Smith Foundation for Health Research (MSFHR); grant support to LM from Canadian Institute of Health Research (CIHR) and Fonds de la Recherché en Santé du Québec; CFK from the National Institutes of Health (NIH) ND-BRIN; GI from Human Early Learning Partnership (HELP); JAB from the Social Sciences and Humanities Research Council of Canada (SSHRC); and AK from the Human Frontier Science Program (HFSP), NSERC, MSFHR, and HELP. We would also like to thank Michael Platt and George R. Mangun for their valuable comments and help with the manuscript.

\section{References}

[1] T. Allison, A. Puce, G. McCarthy, Social perception from visual cues: role of STS region, Trends Cogn. Sci. 4 (2000) 267-278.

[2] S. Baron-Cohen, Mindblindness: An Essay on Autism and Theory of Mind, MIT Press, Massachusetts, 1995.

[3] K. Chawarska, A. Klin, F. Volkmar, Automatic attention cueing through eye movement in 2-year old children with autism, Child Dev. 74 (2003) $1108-1122$.

[4] R.O. Deaner, M.L. Platt, Reflexive social attention in monkeys and humans, Curr. Biol. 13 (2003) 1609-1613.

[5] J. Driver, G. Davis, P. Ricciardelli, P. Kidd, E. Maxwell, S. BaronCohen, Shared attention and the social brain: gaze perception triggers automatic visuo-spatial orienting in adults, Vis. Cogn. 6 (1999) 509-540.

[6] C.K. Friesen, A. Kingstone, The eyes have it! Reflexive orienting is triggered by nonpredictive gaze, Psychon. Bull. Rev. 5 (1998) $490-495$. 
[7] C.K. Friesen, J. Ristic, A. Kingstone, Attentional effects of counterpredictive gaze and arrow cues, J. Exp. Psychol. Hum. Percept. Perform. 30 (2004) 319-329.

[8] D.J. Grelotti, I. Gauthier, R. Schultz, Social interest and the development of cortical face specialization: what autism teaches us about face processing, Dev. Psychobiol. 40 (2002) 13-25.

[9] E.A. Hoffman, J.V. Haxby, Distinct representations of eye gaze and identity in the distributed human neural system for face perception, Nat. Neurosci. 3 (2000) 80-84.

[10] A. Klin, W. Jones, R. Schultz, F. Volkmar, D. Cohen, Visual fixation patterns during viewing of naturalistic social situations as predictors of social competence in individuals with autism, Arch. Gen. Psychiatry 59 (2002) 809-816.

[11] L.G. Klinger, G. Dawson, Prototype formation in autism, Dev. Psychopatol. 13 (2001) 111-124.

[12] A. Kylliäinen, J.K. Hietanen, Attention orienting by another's gaze direction in children with autism, J. Child Psychol. Psychiatry 45 (2004) 435-444.

[13] S.R.H. Langton, V. Bruce, Reflexive visual orienting in response to the social attention of others, Vis. Cogn. 6 (1999) 541-567.
[14] C. Lord, M. Rutter, A. Le Couteur, Autism Diagnostic InterviewRevised: a revised version of a diagnostic interview for caregivers of individuals with possible pervasive developmental disorders, J. Autism Dev. Disord. 24 (1994) 659-685.

[15] C. Lord, S. Risi, L. Lambrecht, et al., The Autism Diagnostic Observations Schedule-Generic: a standard measure of social and communication deficits associated with the spectrum of autism, J. Autism Dev. Disord. 30 (2000) 205-223.

[16] J. Ristic, C.K. Friesen, A. Kingstone, Are eyes special? It depends on how you look at it, Psychon. Bull. Rev. 9 (2002) 507-513.

[17] A. Senju, Y. Tojo, H. Dairoku, T. Hasegawa, Reflexive orienting in response to eye gaze and an arrow in children with and without autism, J. Child Psychol. Psychiatry 45 (2004) 445-458.

[18] R.T. Schultz, et al., Abnormal ventral temporal cortical activity during face discrimination among individuals with autism and Asperger's syndrome, Arch. Gen. Psychiatry 57 (2000) 331-340.

[19] J. Swettenham, A. Condie, R. Campbell, E. Milne, M. Coleman, Does the perception of moving eyes trigger reflexive visual orienting in autism? Philos. Trans. R. Soc. Lond., B Biol. Sci. 358 (2003) $325-334$ 